\title{
FAUNA EDÁFICA EM AGROECOSSISTEMA SEMIÁRIDO COM 17 ANOS DE POUSIO
}

Francisca Irislândia Viana Alves ${ }^{1}$, Ana Ruth Viana Lima ${ }^{2}$, Jamili Silva Fialho ${ }^{3}$

${ }^{1}$ Graduada em Ciências Biológicas da Faculdade de Educação, Ciências e Letras do Sertão Central, FECLESC, Universidade Estadual do Ceará, UECE, Quixadá - CE (irislandiaviana21@gmail.com).

${ }^{2}$ Graduada em Ciências Biológicas da Faculdade de Educação, Ciências e Letras do Sertão Central, FECLESC, Universidade Estadual do Ceará, UECE, Quixadá - CE.

${ }^{3}$ Biológa, Prof ${ }^{a}$. Adjunta, Faculdade Estadual do Ceará, FECLESC, Universidade Estadual do Ceará, UECE, Quixadá - CE.

Recebido em: 03/10/2016 - Aprovado em: 21/11/2016 - Publicado em: 05/12/2016 DOI: 10.18677/EnciBio_2016B 114

\section{RESUMO}

A agricultura tradicional, manejo de corte e queima da vegetação com posterior pousio, pode causar perda de diversidade, erosão até a infertilidade do solo. Por ser um manejo muito utilizado no semiárido pergunta-se: o pousio é suficiente para proporcionar a recomposição do solo? Assim, procurou-se conhecer a comunidade da fauna edáfica em agroecossistema tradicional em pousio há 17 anos (AGRO) comparando com caatinga conservada (CAA). A amostragem ocorreu através da instalação de armadilhas de queda (pitfall-traps). Foram traçados três transectos espaçados em 10 metros com 12 armadilhas que ficaram no campo por sete dias. Os organismos foram identificados em ordem, classe e família e determinadas abundância, riqueza, diversidade e uniformidade das áreas. Os dados foram analisados por meio de análises descritiva e multivariada. Foram amostrados 18.665 indivíduos em 29 táxons com abundância média de 74,07 em AGRO e 24.893 indivíduos, 26 táxons e abundância média de 98,78 na CAA. A caatinga apresentou maior frequência e abundância, pois a estrutura do ambiente conservado permite variadas estratégias de sobrevivência. A diversidade em AGRO foi de 2,70 e uniformidade de 0,555 , enquanto que na CAA foi de 2,43 e 0,518, respectivamente, evidenciando que o pousio favoreceu a recomposição da fauna pela diversificação da cobertura vegetal em sucessão ecológica. Além disso, os colêmbolos, que desempenham importantes funções ambientais, foram mais abundantes nas áreas CAA (Symphypleona) e AGRO (Entomobryomorpha) indicando que o pousio tem possibilitado a restauração do solo. Portanto, o pousio contribui para recuperação do agroecossistema através da recomposição da comunidade da fauna edáfica.

PALAVRAS-CHAVE: agricultura tradicional, bioindicação, meso e macrofauna edáfica.

\section{EDAPHIC FAUNA IN SEMIARID AGROECOSYSTEM UNDER FALLOW FOR 17 YEARS}

\section{ABSTRACT}

Traditional farming, cutting management and burning of vegetation with subsequent fallow, can cause loss of biodiversity, erosion up until soil infertility. It is a widely used 
management in semiarid, is questioned: the fallow is sufficient to provide the recomposition of the soil? So, we tried to know the soil fauna community in traditional agroecosystem with 17 years of follow (AGRO) compared conserved caatinga (CAA). Sampling of organisms occurred by installing pitfall traps. There were three transects spaced in 10 meters with 12 traps were outside the camp for seven days. The organisms were identified in order, class and family and were determined abundance, richness, diversity and uniformity of areas. Data were analyzed using descriptive and multivariate analyzes. Were sampled 18,665 individuals in 29 taxons and mean abundance of 74.07 in AGRO and 24,893 individuals, 26 taxa and mean abundance of 98.78 in the CAA. The caatinga showed higher frequency and abundance, because the structure of the conserved environment allows taxa varied survival strategies. The diversity in AGRO was 2.70 and uniformity of 0.555 , while on the CAA was 2.43 and 0.518 , respectively, showing that fallow favored the restoration of the fauna through the diversification of vegetation in ecological succession. In addition, springtails, which have important environmental benefits were most abundant in the CAA (Symphypleona) and AGRO (Entomobryomorpha) indicating that the fallow has enabled the soil restoration. Therefore, aside contributes to recovery of the agricultural ecosystem through the restoration of soil fauna community.

KEYWORDS: traditional cropping, bioindication, edaphic meso and macrofauna.

\section{INTRODUÇÃO}

No Brasil, o surgimento de áreas degradadas decorrentes de manejos insustentáveis tem sido crescente, causando diversos problemas ao solo, devido à remoção da camada superficial, incluindo a matéria orgânica e, portanto, dos nutrientes (OLIVEIRA \& SOUTO, 2011). Na região semiárida brasileira, o manejo da agricultura tradicional, corte e queima da vegetação para posterior cultivo de sequeiro, desestabiliza a ciclagem da matéria orgânica, causando perda de nutrientes do sistema solo-vegetação, além de processos erosivos e degradação do solo (PEDROSO JÚNIOR et al., 2008). Além disso, a caatinga, vegetação dominante dessa região, é manejada de forma inadequada pelo agricultor que utiliza técnicas muitas vezes agressivas ao solo, comprometendo assim sua qualidade (NUNES et al., 2009). Em se tratando das limitações impostas pelos fatores ambientais, como amplo período anual de seca, é um ecossistema facilmente degradável e de difícil recuperação (NUNES et al., 2006), sendo conhecido como representante de um dos ecossistemas mais vulneráveis à desertificação (ARAÚJO \& SOUZA, 2011).

No sistema agrícola ou agroecossistema, o solo é um recurso precioso e o manejo adotado pode conservá-lo (DEVIDE \& CASTRO, 2008), contribuindo para a sua fertilidade, o aumento da qualidade nutricional e podendo tornar possível a colonização de habitats antes desfavoráveis (LIMA et al., 2010), como também pode degradá-lo, ocasionando erosão, perda da matéria orgânica e dos nutrientes (DEVIDE \& CASTRO, 2008). A ação antrópica através das práticas agrícolas interfere na fauna edáfica que utiliza o agroecossistema como habitat (LAVELLE et al., 1997), pois o uso intensivo do sistema pode modificar o equilíbrio (CORREIA et al., 2009) e alterar o abastecimento de alimento, a formação de microambientes e as competições intra e interespecífica (ALVES, 2006) afetando, consequentemente, a diversidade dos invertebrados da fauna edáfica (BARETTA et al., 2003; CORREIA et al., 2009; NUNES et al., 2009). 
A fauna edáfica que, compreende uma expressiva parcela de organismos que têm como habitat o solo ou que passam uma ou mais fases da vida nele (ASSAD, 1997), é utilizada como importante indicador biológico da qualidade do solo, por sua atuação nos processos biológicos dos ecossistemas, mostrando eficácia inclusive na avaliação de agroecossistemas degradados (WINK et al., 2005; ARAÚJO et al., 2009). Além disso, a fauna apresenta importante função na sustentabilidade do ecossistema devido aos seus efeitos no solo, uma vez que a composição da comunidade dos invertebrados pode refletir no padrão de funcionamento deste (OLIVEIRA \& SOUTO, 2011), por promover uma regulação dos processos de decomposição da matéria orgânica e, consequentemente, da liberação de nutrientes (CORREIA, 2002).

A sensibilidade da fauna edáfica às modificações ambientais é fácil e economicamente viável de medir, auxiliando na tomada de decisões em agroecossistemas que buscam um manejo mais sustentável (MANHÃES, 2011), contribuindo na avaliação da sustentabilidade de qualquer ambiente (ANDRADE, 2000; MANHÃES, 2011) e possibilitando a percepção de seu estado atual e de modificações causadas por forças internas e externas (bióticas e abióticas) que ocorrem ao longo do tempo (MELO et al., 2009).

Ao estudarem a fauna edáfica em solos submetidos a diferentes sistemas de manejo no semiárido brasileiro NUNES et al. (2009) verificaram que os sistemas que sofreram queimadas para plantio sofreram diminuição das populações de diferentes espécies, além das formas juvenis (larvas), e ausência de grupos. O mesmo foi observado por LIMA et al. (2010), em agroecossistemas com manejo de corte e queima da vegetação, pela redução da densidade e da riqueza da macrofauna edáfica.

Assim, considerando que a queimada da vegetação como prática agrícola acarreta efeitos negativos sobre as populações de invertebrados edáficos, como eliminação da serapilheira e consequentemente, desestrutura do habitat (CORREIA \& OLIVEIRA, 2000), pergunta-se: o pousio, após manejo tradicional, favorece a recomposição da fauna do solo? Portanto, objetivou-se conhecer a fauna edáfica em agroecossistema tradicional de corte e queima no semiárido, em pousio há aproximadamente 17 anos.

\section{MATERIAL E MÉTODOS}

A pesquisa foi realizada na Fazenda Não Me Deixes, localizada no distrito de Daniel de Queiroz, município de Quixadá, Ceará. A propriedade possui 929 ha e em 1998 teve 300 ha reconhecidos como RPPN pelo Instituto Brasileiro do Meio Ambiente e dos Recursos Renováveis (IBAMA). A fazenda se situa a 4\%49'34" S e 3858'9" W, com altitude média de $210 \mathrm{~m}$. Apresenta clima tropical quente semiárido, com temperatura média anual entre $26^{\circ}$ a $28^{\circ} \mathrm{C}$, pluviosidade anual de $838,1 \mathrm{~mm}$, com estação seca de maio à janeiro e estação chuvosa concentrada nos meses de fevereiro a abril, vegetação de Caatinga Arbustiva Densa, Caatinga Arbustiva Fechada e Floresta Caducifólia Espinhosa e solos das classes Luvissolos, Argissolos, Planossos e Neossolos Litólicos (CEARÁ, 2015).

A fauna edáfica foi coletada em duas áreas: um agroecossistema tradicional (AGRO), que utilizou corte e queima da vegetação para posterior plantio de milho e mamona, sob pousio há aproximadamente 17 anos, e uma área de caatinga conservada (CAA), adjacente ao agroecossistema, no mês de junho de 2013. A captura da meso e macrofauna edáfica ocorreu através da instalação de armadilhas de queda ("pitfall-traps"), espaçadas em $10 \mathrm{~m}$ (em média 1 armadilha por $\mathrm{m}^{2}$ ) e 
durante 7 dias, para amostrar maior diversidade de táxons (AQUINO et al., 2006). Ao longo de cada área analisada foram traçadas três linhas (transectos), espaçadas em $10 \mathrm{~m}$, onde foram distribuídas 12 armadilhas, totalizando 36 armadilhas por área (Figura 1). As armadilhas, frascos de plástico com $10 \mathrm{~cm}$ de diâmetro e $20 \mathrm{~cm}$ de profundidade, foram colocadas ao nível do solo e preenchidas até $1 / 3$ de sua capacidade com líquido conservante (álcool 53\% e duas gotas de detergente para quebrar a tensão superficial da água). Após desinstaladas foram levadas para o laboratório, tendo seu conteúdo transferido para solução conservante de álcool $70 \%$. O conteúdo dos frascos foi vertido em placa de Petri e os invertebrados observados sob microscópio estereoscópico para identificação (AQUINO et al., 2006).

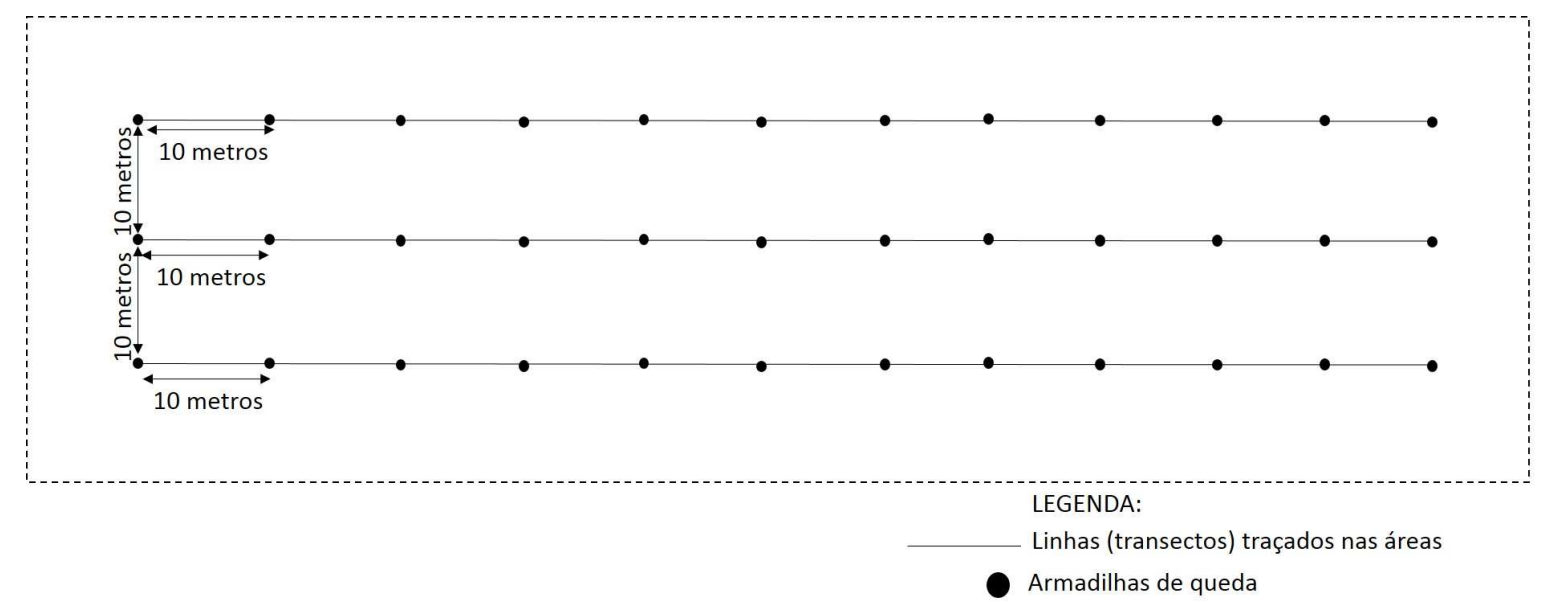

FIGURA 1 - Croqui de distribuição das armadilhas de queda nas áreas de agroecossistema e caatinga, Fazenda Não Me Deixes, distrito de Daniel de Queiroz, Quixadá- CE.

A partir dos resultados foram definidos o número total de indivíduos, a abundância média dos táxons em número de indivíduos por armadilha por dia (indivíduos armadilha ${ }^{-1}$ dia $^{-1}$ ); a riqueza, que corresponde ao número de táxons identificados, a diversidade e a uniformidade da área (MOÇO et al., 2005; SOUZA et al., 2008). Os táxons que apresentaram abundância média inferior a 0,5, nas duas áreas, foram agrupados em outros. A diversidade da área, que expressa a relação entre o número de táxons e a distribuição do número de indivíduos entre os táxons, foi determinada utilizando-se o Índice de Diversidade de Shannon-Weaver $\left(H^{\prime} ; 1\right)$, indicado para cálculos de diversidade da biologia do solo por considerar a riqueza das espécies/táxons e sua abundância relativa (MAGURRAN, 2004) e definido por:

$$
\begin{aligned}
& \mathrm{H}^{\prime}=-\square \text { pi . logpi } \\
& \text { Onde pi = ni/N; } \\
& \text { ni = valor de importância da cada espécie ou táxon; } \\
& \quad \mathrm{N}=\text { total dos valores de importância. }
\end{aligned}
$$

A uniformidade da área foi calculada de acordo com o Índice de Pielou (e; 2), que representa o padrão de distribuição dos indivíduos entre as espécies ou táxons (MAGURRAN, 2004), e é definido por:

$$
\mathrm{e}=\mathrm{H}^{\prime} / \log \mathrm{S}
$$

em que H' =Índice de Shannon;

$S$ = Número total de espécies/táxons. 
Os dados foram analisados por meio de análises descritivas, medidas de posição (média) e de variabilidade (desvio padrão), e multivariada (análise dos componentes principais).

\section{RESULTADOS E DISCUSSÃO}

O número total de indivíduos capturados no agroecossistema em pousio foi de 18.665 e na caatinga conservada de 24.893 organismos (Tabela 1). Quando a cobertura vegetal é diversificada, como na caatinga conservada, a serrapilheira é mais heterogênea, possibilitando substratos mais diversos em qualidade nutricional e orgânica (NUNES et al., 2012), o que favorece a diversidade da comunidade da fauna edáfica (MOÇO et al., 2005; NUNES et al., 2012). Desta forma, as condições proporcionadas pela serrapilheira dão subsídios para o surgimento de maior número de nichos ecológicos e uma complexa rede alimentar, minimizando a competição e favorecendo a ocorrência dos organismos (LAVELLE et al.,1997; MOÇO et al., 2005; NUNES et al., 2012).

TABELA 1 - Número total de indivíduos, abundância média (indivíduos por armadilha por dia) \pm erro-padrão, riqueza total, diversidade e uniformidade do agroecossistema de corte e queima sob pousio (AGRO) e da área de caatinga conservada (CAA), Fazenda Não Me Deixes, Quixadá, CE

\begin{tabular}{lccccc}
\hline Área & $\begin{array}{c}\text { No total de } \\
\text { indivíduos }\end{array}$ & $\begin{array}{c}\text { Abundância } \\
\text { média (Ind. }_{\text {Arm }^{-1} \text { dia }^{-1} \text { ) }}\end{array}$ & $\begin{array}{c}\text { Riqueza } \\
\text { Total }\end{array}$ & $\begin{array}{c}\text { Diversidade } \\
\text { (Índice de } \\
\text { Shannon- } \\
\text { Weaver }\end{array}$ & $\begin{array}{c}\text { Uniformidade } \\
\text { (Índice de } \\
\text { Pielou) }\end{array}$ \\
\hline AGRO & 18.665 & $74,07 \pm 4,22$ & 29 & 2,70 & 0,555 \\
\hline CAA & 24.893 & $98,78 \pm 4,45$ & 26 & 2,43 & 0,518 \\
\hline
\end{tabular}

A abundância média, a riqueza total e os índices de diversidade e uniformidade indicam que o tempo de pousio tem proporcionado a recomposição da fauna edáfica no agroecossistema, provavelmente, pela variedade de microhabitats e de oferta de recursos, advindos da diversidade da vegetação em regeneração (SILVA et al., 2006, 2008); o que implica maior conteúdo de matéria orgânica, tornando o ambiente favorável para os invertebrados edáficos (SILVA et al., 2006). Além disso, os resultados obtidos são semelhantes aos de NUNES et al. (2008) que observaram reocupação pela fauna edáfica de áreas submetidas à queimada, a partir de três anos após fogo.

A diversidade dos organismos comprovou que o pousio, ao possibilitar o desenvolvimento da vegetação, provavelmente, leva a uma maior disponibilidade de nichos ecológicos e consequente recomposição das cadeias alimentares, favorecendo a restauração da qualidade do solo submetido à queimada (NUNES et al., 2006), pois o resultado obtido em AGRO foi maior do que o encontrado na CAA e também do observado por NUNES et al. (2009), ao analisarem a diversidade da fauna do solo no semiárido nordestino em área de caatinga preservada há 50 anos $\left(H^{\prime}=1,09\right)$.

A uniformidade corroborou a importância do pousio para a restauração do agroecossistema tradicional. Quando os resultados são comparados com a caatinga conservada, estudada nessa e em outras pesquisas, como a realizada por NUNES et al., (2009) que obtiveram valores de 0,27; percebe-se que os dados são superiores, ou seja, o período de pousio tem favorecido à uniformidade da área. $\mathrm{O}$ ENCICLOPÉDIA BIOSFERA, Centro Científico Conhecer - Goiânia, v.13 n.24; p. 12302016 
aumento da uniformidade é promovido pela disponibilidade de melhores condições ambientais, favorecendo a reprodução dos invertebrados (BROWN et al., 2004) e pela melhoria na qualidade e na quantidade de resíduos vegetais, proporcionados pela sucessão estabelecida durante o pousio, que servem de alimento e abrigo para os organismos edáficos (BARETTA et al., 2003). A maior uniformidade desse ambiente pode indicar ainda maior equilíbrio na composição dos indivíduos, como observado por ALMEIDA et al. (2009) ao pesquisarem a macrofauna, visto que as condições de pousio podem proporcionar um microclima favorável e recursos alimentares capazes de atrair mais indivíduos de diferentes táxons (DIAS et al., 2006).

O primeiro e o segundo componentes da análise dos componentes principais (ACP) realizada entre as áreas e os táxons explicam $41,67 \%$ da variação total dos dados (Figura 2). As amostras de AGRO se concentram nos quadrantes 1 e 3 e estão sob influência dos táxons larva de Diptera, Auchenorryncha e Hymenoptera. Já as amostras de CAA estão centralizadas e no quadrante 4 sob influência dos táxons Poduromorpha e Symphypleona.

Destaca-se que colêmbolos (Entomobryomorpha) e Hymenoptera (formigas) podem ser bioindicadores de reabilitação do solo (BARETTA et al., 2006). Em se tratando dos colêmbolos, a posição ocupada na cadeia trófica, o papel funcional exercido nos processos que envolvem a dinâmica da matéria orgânica e a sensibilidade em resposta a perturbações podem ser indicativos eficientes para que esse grupo seja considerado bom indicador de qualidade do solo (COLEMAN \& HENDRIX, 2000; ROVEDDER et al., 2009; SILVA et al., 2013).

Os organismos de Entomobryomorpha apresentam significativa função na cadeia alimentar através da promoção de degradação da matéria orgânica no complemento dos ciclos biogeoquímicos (MELO et al., 2009). Quanto às formigas estudos realizados em diferentes agroecossistemas têm comprovado o grande potencial que estes invertebrados edáficos oferecem como bioindicadores de perturbações ambientais, sendo mais frequentes, principalmente, durante 0 processo de recuperação das áreas degradadas (BARETTA et al., 2003; SILVA et al., 2012).

Nos estudos de NUNES et al. (2006) e (2008) observou-se que o grupo Formicidae esteve presente em maiores proporções nos sistemas em pousio e na mata nativa. Além disso, os valores de abundância média de Formicidae (Tabela 2) podem indicar de que o sistema está em equilíbrio, uma vez que a importância desse grupo para a comunidade da fauna edáfica está relacionada ao hábito social e a repartição do trabalho, que são atribuídos à sua atividade, onde há a construção de ninhos, utilizando as partículas minerais do solo, matéria orgânica, secreções e dejetos, considerados de relevante importância para que ocorram os processos de decomposição em ecossistemas tropicais (NUNES et al., 2006, 2008). 


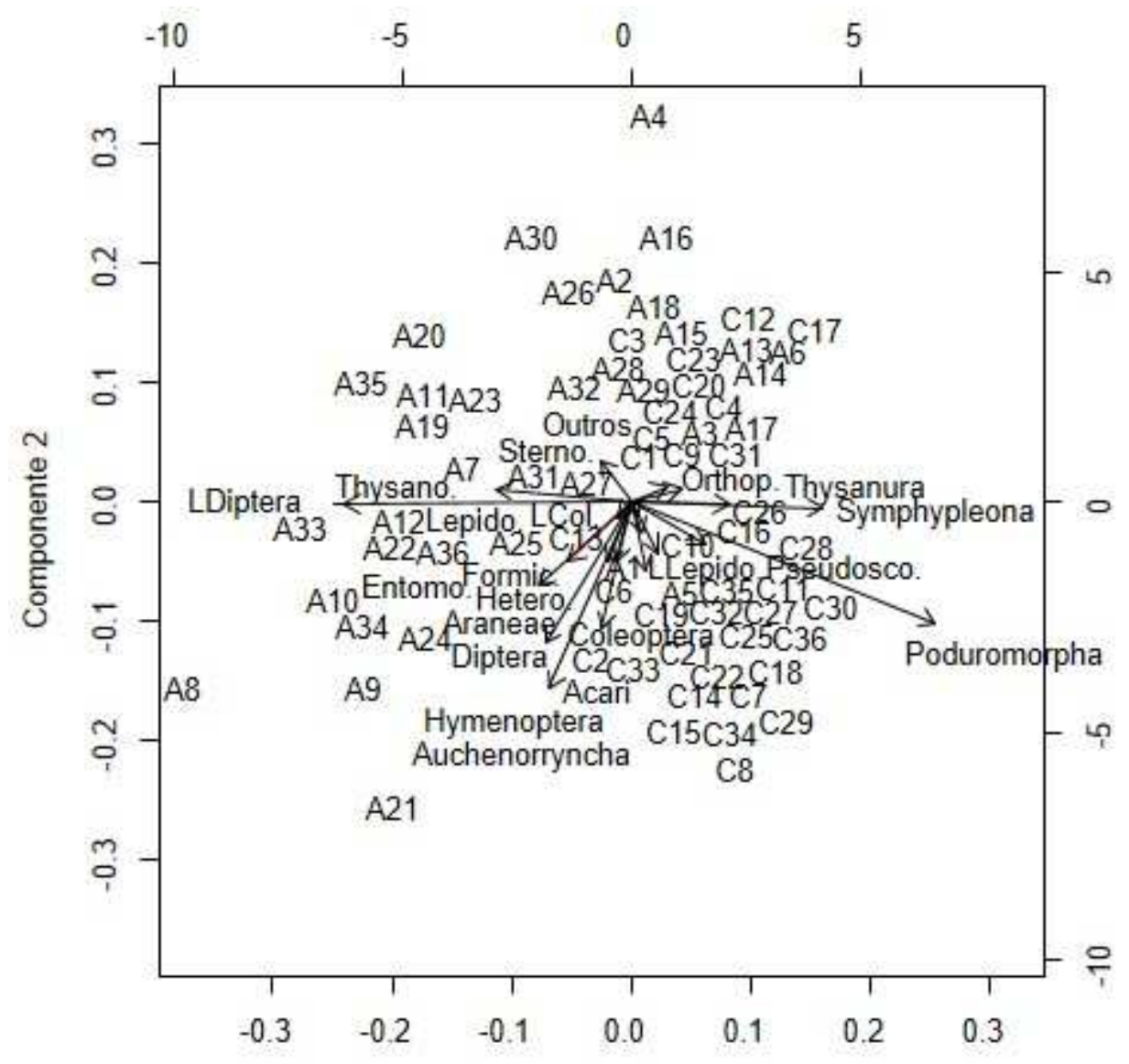

Componente 1

FIGURA 2 - Distribuição da nuvem das áreas e táxons da fauna edáfica relativas aos componentes principais 1 e 2. LDiptera larva de Diptera, Entomo. Entomobryomorpha, Sterno. Sternorryncha, Thysano. Thysanoptera, Lepido. Lepidoptera, Hetero. Heteroptera, Orthop. Orthoptera, Pseudosco. Pseudoscorpionida, LLepido. larva de Lepidoptera, Formic. Formicidae, LCol. larva de Coleoptera, A 1 a 36 armadilhas do agroecossistema e $\mathrm{C} 1$ a 36 armadilhas da caatinga.

Observa-se ainda que os táxons Poduromorpha, larva de Diptera, Symphypleona e Auchenorryncha estão próximos à superfície do gráfico da ACP, indicando que são mais representativos em relação aos outros, que estão mais afastados. Esse resultado pode ser confirmado com os valores de importância dos táxons na formação dos componentes principais, sendo Poduromorpha e larva de Diptera representativos na formação do componente 1 e larva de Diptera, Auchenorryncha e Symphypleona do componente 2, com acréscimo de Entomobryomorpha (Tabela 2).

Os colêmbolos, representados pelas subordens Symphypleona e Entomobryomorpha e mais abundantes nas áreas CAA e AGRO, respectivamente, apresentam importante função detritívora e fungívera, através das quais contribuem para a decomposição da matéria orgânica e controle das populações de microrganismos, principalmente dos fungos (MELO et al., 2009), indicando, portanto, que o pousio tem possibilitado a recomposição biológica do solo. 
TABELA 2 - Abundância média (AM), desvio padrão (DP) e valor de importância dos táxons da fauna do solo na formação dos componentes 1 (C1) e 2 (C2) da Análise dos Componentes Principais (ACP), $n=36$, na área do agroecossistema de corte e queima sob pousio (AGRO) e da caatinga conservada (CAA), Fazenda Não Me Deixes, Quixadá - CE

\begin{tabular}{lcccccc}
\hline Táxons & AGRO & \multicolumn{2}{c}{ CAA } & \multicolumn{3}{c}{ Valor de Importância } \\
& AM & DP & AM & DP & C1 & C2 \\
\hline Acari & 108,2 & 72,7 & 136,6 & 78,8 & $-0,371$ & 0,174 \\
Araneae & 4,6 & 3,0 & 4,0 & 2,7 & $-0,12$ & $-0,172$ \\
Auchenorryncha & 4,2 & 4,9 & 3,8 & 2,9 & $-0,155$ & $-\mathbf{0 , 5 4 9}$ \\
Coleoptera & 5,6 & 3,5 & 6,2 & 3,0 & $-0,198$ & 0,179 \\
Diptera & 20,3 & 14,2 & 15,6 & 8,5 & $-0,175$ & $-0,242$ \\
Entomobryomorpha & 76,7 & 55,5 & 56,4 & 23,5 & $-0,174$ & $-\mathbf{0 , 5 8 1}$ \\
Formicidae & 57,8 & 33,3 & 58,7 & 19,7 & $-0,184$ & 0,218 \\
Heteroptera & 0,7 & 0,8 & 1,1 & 3,1 & $-0,175$ & $-0,467$ \\
Hymenoptera & 3,7 & 3,9 & 2,8 & 2,1 & $-0,163$ & $-0,416$ \\
Larva Coleoptera & 1,2 & 1,2 & 1,2 & 1,4 & 0,113 & 0,266 \\
Larva Diptera & 9,3 & 13,1 & 2,8 & 5,5 & $-\mathbf{0 , 5 4 3}$ & $\mathbf{0 , 5 9 1}$ \\
Larva Lepidoptera & 0,3 & 0,6 & 0,7 & 0,8 & $-0,15$ & $-0,153$ \\
Lepidoptera & 0,8 & 1,0 & 0,6 & 0,9 & 0,105 & $-0,185$ \\
Orthoptera & 2,1 & 2,1 & 2,3 & 1,4 & $-0,271$ & 0,259 \\
Poduromorpha & 16,4 & 17,5 & 73,6 & 36,3 & $\mathbf{0 , 5 7 4}$ & $-0,358$ \\
Pseudoscorpionida & 0,5 & 0,9 & 1,3 & 1,3 & 0,14 & $-0,127$ \\
Sternorryncha & 4,6 & 4,3 & 4,3 & 2,8 & 0,114 & $-0,273$ \\
Symphypleona & 195,8 & 149,3 & 316,5 & 161,6 & 0,363 & $-\mathbf{0 , 5 3 4}$ \\
Thysanoptera & 2,1 & 3,2 & 0,3 & 0,6 & $-0,258$ & $-0,186$ \\
Thysanura & 0,8 & 1,1 & 1,6 & 1,2 & 0,185 & $-0,137$ \\
Outros & 1,8 & 1,8 & 1,2 & 1,9 & 0,122 & 0,442 \\
\hline
\end{tabular}

Acari (ácaros) esteve presente em grande quantidade tanto no AGRO quanto no CAA. Eles constituem um dos mais variados grupos de artrópodes, que contribuem de maneira significativa para o funcionamento de ecossistemas, e alimentam-se de uma enorme diversidade de substratos, podendo ser classificados de acordo com suas funções em fitófagos, predadores e fungívoros (FLECHTMAN, 1985). Esses organismos exercem funções que melhoram o solo, principalmente quando se trata da mobilização de nutrientes (PRIMAVESI, 2002; ARAÚJO et al., 2009). Além disso, desempenham papel de extrema importância para os processos edáficos, tais como: ciclagem de nutrientes, decomposição da matéria orgânica, melhoria de atributos físicos como agregação, porosidade, infiltração de água, e no funcionamento biológico do solo (SANGINGA et al., 1992; ARAÚJO et al., 2009), controlando, desta forma, as populações de outros organismos no solo, especialmente no que diz respeito à microbiota (MELO et al., 2009).

Estudo realizado por VICENTE et al., (2010) quando avaliavam fauna edáfica em recuperação de áreas degradadas em Minas Gerais, confirmou a presença de ácaros em ambientes equilibrados, onde havia maior teor de matéria orgânica e umidade. Sendo, portanto um táxon indicador de locais em restauração e 
contribuindo para a confirmação dos benefícios do pousio para a reabilitação da fauna do solo.

\section{CONCLUSÕES}

O pousio do agroecossistema tem contribuído para a restauração do habitat e, consequentemente, da fauna edáfica, uma vez que tem proporcionado aumento da abundância, riqueza, diversidade e uniformidade do agroecossistema. Além disso, as abundâncias, dos táxons Acari, Entomobryomorpha, Formicidae e Symphypleona, semelhantes entre o agroecossistema e a caatinga conservada corroboram a restauração, visto que são bioindicadores de recuperação ambiental.

\section{AGRADECIMENTOS}

Ao Conselho Nacional de Desenvolvimento Científico e Tecnológico (CNPq) pela bolsa de iniciação científica concedida.

\section{REFERÊNCIAS}

ALMEIDA, M. V. R. de.; OLIVEIRA, T. S. de.; BEZERRA, A. M. E. Biodiversidade em sistemas agroecológicos no município de Choró, CE, Brasil. Ciência Rural, Santa Maria, v. 39, n. 4, p. 1080-1087, 2009. Disponível em: < http://dx.doi.org/10.1590/S0103-84782009005000047>. doi: 10.1590/S010384782009005000047.

ALVES, M. V.; BARETTA, D.; CARDOSO, E. J. B. N. Fauna edáfica em diferentes sistemas de cultivo no estado de São Paulo. Revista de Ciências Agroveterinárias, Lages, v. 5, n. 1, p. 33-43, 2006. Disponível em: <http://dx.doi.org/10.1590/S1983-40632013000200001>. doi: 10.1590/S198340632013000200001.

ANDRADE, L. B. de. O uso da fauna edáfica como bioindicadora de modificações ambientais em áreas degradadas, 2000, 51 f. Monografia (Bacharel em Ecologia), Universidade Federal Rural do rio de Janeiro - UFRRJ, 2000. Disponível em: <http://www.ufrrj.br/institutos/if/pdf/monografia06.pdf>.

AQUINO, A. M.; AGUIAR-MENEZES, E. L.; QUEIROZ, J. M. Recomendações para coleta de artrópodes terrestres por armadilhas de queda (pitfall-traps). Circular Técnica no 18, Embrapa Agrobiologia, Seropédica, 8 p., 2006. Disponível em: <https://www.infoteca.cnptia.embrapa.br/bitstream/doc/628430/1/cit018.pdf>. doi: 1519-7328.

ARAÚJO, C. S. F.; SOUSA, A. N. Estudo do processo de desertificação na Caatinga: uma proposta de educação ambiental. Ciências e Educação, vol. 17, n. 4, 2011. Disponível em: <http://www.redalyc.org/articulo.oa?id=251021295013>. doi: 10.1590/S1516-73132011000400013.

ARAÚJO, K. D.; PARENTE, H. N.; CORREIA, K. G.; RODRIGUES, M. Q.; DANTAS, R. T.; ANDRADE, A. P. de.; SOUTO, J. S. Influência da precipitação pluvial sobre a mesofauna invertebrada do solo em área de caatinga no semiárido da Paraíba. Revista Eletrônica do Curso de Geografia, Campos Jataí, n. 12, 12 p., 2009. 
Disponível em: <http://dx.doi.org/10.5216/rev.\%20geoambie.v0i12.25979>. doi: 10.5216/rev.\%20geoambie.v0i12.25979.

ASSAD, M. L. L. Fauna do solo. In: VARGAS, M. A. T.; HUNGRIA, M. Biologia dos solos do cerrado. Embrapa Cerrados, Planaltina, p. 363-443, 1997. Disponível em: <http://livimagens.sct.embrapa.br/amostras/00063220.pdf>. doi: 00063220.

BARETTA, D.; SANTOS, J. C. P.; BERTOL, I.; ALVES, M. V.; MANFOI, A. F.; BARETTA, C. R. D. M. Efeito do cultivo do solo sobre a diversidade da fauna edáfica no planalto sul catarinense. Revista de Ciências Agroveterinárias, Lages, v. $5, \quad$ n. 2, p. 108-117, 2006. Disponível em < http://rca.cav.udesc.br/rca_2006_2/artigo_baretta.pdf>. doi: 1676-9732.

BARETTA, D.; SANTOS, J. C. P.; MAFRA, A. L.; WILDNER, L. do P.; MIQUELIUTI, D. J. Fauna edáfica avaliada por armadilhas e catação manual afetada pelo manejo do solo na região Oeste Catarinense. Revista de Ciências Agroveteriárias, Lages, v. 2, n. 2, p. 97-106, 2003. Disponível em: <http://intranetdoc.epagri.sc.gov.br/producao_tecnico_cientifica/DOC_9863.pdf>.doi: 2238-1171.

BROWN, G. G.; MORENO, A. G.; BAROIS, I.; FRAGOSO, C.; ROJAS, P.; HERNÁNDEZ, B.; PATRÓN, J. C. Soil macrofauna. In: SE MEXICAN PASTURES AND THE EFFECT OF CONVERSION FROM NATIVE TO INTRODUCED PASTURES. Agriculture, Ecosystems and Environment, v. 103, p. 313-327, 2004. Disponível em: <http://www.sciencedirect.com/science/journal/01678809>. doi: 10.1016/j.agee.2003.12.006.

CEARÁ. Governo do Estado do Ceará. Secretaria do Planejamento e Gestão. Instituto de Pesquisa e Estratégia Econômica. O Perfil Básico Municipal, Flávio Ataliba Flexa Daltro Barreto, 18 p., 2015. Disponível em: <http://www.ipece.ce.gov.br/publicacoes/perfil_basico/pbm-2015/Quixada.pdf>.

COLEMAN, D. C.; HENDRIX, P. F. Invertebrates as webmasters in ecosystems. London: CABI Publishing, 336 p., 2000. Disponível em: <http://coweeta.uga.edu/publications/1219.pdf>. doi: 085199394X.

CORREIA, K. G.; ARAUJO, K. D.; AZEVEDO, L. G. de.; BARBOSA, E. A.; SOUTO, J. S.; SANTOS, T. S. Macrofauna edáfica em três diferentes ambientes na região do Agreste Paraibano, Brasil. Engenharia Ambiental, Espírito Santo do Pinhal, v. 6, n. 1, p. 206-213, 2009. Disponível em:

<http://dx.doi.org/10.18188/19831471/sap.v15n1p94-99>. doi: 10.18188/19831471/sap.v15n1p94-99.

CORREIA, M. E. F. Potencial de utilização dos atributos das comunidades de fauna de solo e de grupos chave de invertebrados como bioindicadores do manejo de ecossistemas. Documento no 157, Embrapa Agrobiologia, Seropédica, 14 p., 2002. Disponível em: <http://www.infoteca.cnptia.embrapa.br/handle/doc/624781>. doi: 1517-88498. 
CORREIA, M. F. E.; OLIVEIRA, L. C. M. de. Fauna de solo: Aspectos gerais e metodológicos. Documento no 112, Embrapa Agrobiologia, Seropédica, 48 p., 2000. Disponível em: <http://ainfo.cnptia.embrapa.br/digital/bitstream/CNPAB2010/27350/1/doc112.pdf>. doi: 1517-8498.

DEVIDE, A. C. P.; CASTRO, C. M. de. Manejo do solo e a dinâmica da fauna edáfica. Pesquisa \& Tecnologia, v. 5, n. 2, 7 p., 2008. Disponível em: < http://www.aptaregional.sp.gov.br/Pesquisa-Tecnologia/pesquisa-etecnologia.html?thanks=enquete $>$. doi: 2316-5146.

DIAS, P. F.; SOUTO, S. M.; CORREIA, M. E. F.; ROCHA, G. P.; MOREIRA, J. F.; RODRIGUES, K. de M.; FRANCO, A. A. Árvores fixadoras de nitrogênio e macrofauna do solo em pastagem de híbrido de Digitaria. Pesquisa Agropecuária Brasileira, Brasília, v. 41, n. 6, p. 1015-1021, 2006. Disponível em: <http://dx.doi.org/10.1590/S0100-204X2006000600018>. doi: 10.1590/S0100204X2006000600018.

FLECHTMAN, C. H. W. Ácaros de importância agrícola. São Paulo: Nobel, 189 p., $1985 . \quad$ Disponível em: <https://www.bdpa.cnptia.embrapa.br/busca?b=ad\&id=210714\&biblioteca=vazio\&bu sca=autoria:\%22FLECHTMANN,\%20C.\%20H.\%20W.\%22\&qFacets=autoria:\%22FLE CHTMANN,\%20C.\%20H.\%20W.\%22\&sort=\&paginacao=t\&paginaAtual=1 >. $\quad$ doi: $12126-1$.

LAVELLE, P.; BIGNELL, D.; LEPAGE, M.; WOLTERS, V.; ROGER, P.; INESON, P.; HEAL, O. W.; DHILLION, S. Soil function in a cganging word: the role of invertebrate ecosystem engineers. European Journal of Soil Biology, v. 33, p. 159-193, 1997. Disponível em < http://pierre-armand-roger.fr/publications/pdf/194_lavelle.pdf>. doi: 35400007052344.0010.

LIMA, S. S. de.; AQUINO, A. M. de.; LEITE, L. F. C.; VELÁSQUEZ, E.; LAVELLE, P. Relação entre macrofauna edáfica e atributos químicos do solo em diferentes agroecossistemas. Pesquisa Agropecuária Brasileira, Brasília, v. 45, n. 3, p. 322331, 2010. Disponível em: <http://www.alice.cnptia.embrapa.br/handle/doc/858646>. doi: 858646.

MAGURRAN, A. E. Measuring biological diversity. Blackwell, Oxford, 256 p., 2004. Disponível em: <http://www2.ib.unicamp.br/profs/thomas/NE002_2011/maio10/Magurran\%202004\% 20c2-4.pdf>. doi: 978-0-632-05633-0.

MANHÃES, C. M. C. Caracterização da fauna edáfica de diferentes coberturas vegetais no Norte do Estado do Rio de Janeiro, Brasil, 2011, 71 f. Dissertação (Mestrado em Produção Vegetal), Universidade Estadual do Norte Fluminense Darcy Ribeiro - UENE, Campos dos Goytacazes - RJ, 2011. Disponível em: <http://www.uenf.br/Uenf/Downloads/PRODVEGETAL_3434_1303332500.pdf>.

MELO, F. V. de.; BROWN, G. G.; CONSTANTINO, R.; LOUZADA, J. N. C.; LUIZÃO, F. J.; MORAIS, J. W. de.; ZANETTI, R. A importância da meso e macrofauna do solo 
na fertilidade e como biondicadores. Boletim Informativo da SBCS, 3 p., 2009. Disponível em: <https://www.alice.cnptia.embrapa.br/alice/handle/doc/428233>. doi: 428233.

MOÇO, M. K. da S.; GAMA-RODRIGUES, E. F. da.; GAMA-RODRIGUES, A. C. da.; CORREIA, M. E. F. Caracterização da fauna edáfica em diferentes coberturas vegetais na região Norte Fluminense. Revista Brasileira Ciência do Solo, 10 p., 2005. Disponível em: <http://dx.doi.org/10.1590/S0100-06832005000400008>. doi: 10.1590/S0100-06832005000400008.

NUNES, L. A. P. L.; SILVA, D. I. B. da.; ARAÚJO, A. S. F. de.; LEITE, L. F. C.; CORREIA, M. E. F. Caracterização da fauna edáfica em sistemas de manejo para produção de forragens no Estado do Piauí. Revista Ciência Agronômica, v. 43, n. 1 , p. 30-37, 2012. Disponível em: <http://dx.doi.org/10.1590/S180666902012000100004>. doi: 10.1590/S1806-66902012000100004.

NUNES, L. A. P. L.; ARAÚJO FILHO, J. A. de.; MENEZES, R. Í. de Q. Diversidade da fauna edáfica em solos submetidos a diferentes sistemas de manejo no semiárido nordestino. Scientia Agrária, Curitiba, v. 10, n. 1, p. 043-049, 2009. Disponível em: $<$ revistas.ufpr.br/agraria/article/download/13162/9881>.

10.5380/rsa.v10i1.13162.

NUNES, L. A. P. L.; ARAÚJO FILHO, J. A. de.; MENEZES, R. Í. de Q. Recolonização da fauna edáfica em áreas de Caatinga submetidas a queimadas. Revista Caatinga (Mossoró, Brasil), v. 21, n. 3, p. 214-220, 2008. Disponível em: <periodicos.ufersa.edu.br/revistas/index.php/sistema/article/download/375/402>. doi: $375 / 402$.

NUNES, L. A. P. L.; ARAÚJO FILHO, J. A. de.; MENEZES, R. Í. de Q. Impacto da queimada e do pousio sobre a qualidade de um solo sob Caatinga no Semiárido Nordestino. Revista Caatinga, Mossoró, Brasil, v. 19, n. 2, p. 200-208, 2006. Disponível em: <http://www.alice.cnptia.embrapa.br/bitstream/doc/855782/1/APIImpactodaqueimada .pdf>. doi: 855782/1.

OLIVEIRA, E. M. de.; SOUTO, J. S. Mesofauna edáfica como indicadora de áreas degradadas. Revista Verde, Pombal - PB - Brasil, v. 6, n. 1, p 01-09, 2011. Disponível em: < http://dx.doi.org/10.18378/rvads.v6i1.423>. doi: 10.18378/rvads.v6i1.423.

PEDROSO JÚNIOR, N. N.; MURRIETA, R. S. S., ADAMS, C. A agricultura de corte e queima: um sistema em transformação. Boletim do Museu Paraense Emílio Goeldi, Ciências Humanas, v. 3, n. 2, p. 153-174, 2008. Disponível em: <http://producao.usp.br/handle/BDPI/2659>. doi: 1981-8122.

PRIMAVESI, A. Manejo ecológico do solo: a agricultura em regiões tropicais. São Paulo: Nobel, $541 \quad$ p., $2002 . \quad$ Disponível em: <https://books.google.com.br/books/about/Manejo_ecol\%C3\%B3gico_do_solo_a_ag ricultura.html?hl=pt-BR\&id=DHo2zLdESkEC>. doi: 85-213-0004-2. 
ROVEDDER, A. P.; ANTONIOLLI, Z. I.; SPAGNOLLO, E.; VENTURINI, S. F. Fauna edáfica em solo suscetível à arenização na região sudoeste do Rio Grande do Sul. Revista de Ciências Agroveterinárias, Lages, v. 3, n. 2, p. 87-96, 2004. Disponível em: <http://rca.cav.udesc.br/rca_2004_2/rovedder.pdf>. doi: 1676-9732.

SANGINGA, N.; MULONGOY, K.; SWIFT, M. J. Contribution of soil organisms to the sustainability and productivity cropping systems in the tropics. Agriculture, Ecosystem and Environment, v. 41, p. 135-152, 1992. Disponível em: <https://www.bdpa.cnptia.embrapa.br/consulta/busca?b=ad\&id=647294\&biblioteca $=$ v azio\&busca=autoria:\%22SANGINGA,\%20N.\%22\&qFacets=autoria:\%22SANGINGA, $\% 20 \mathrm{~N} . \% 22 \&$ sort=\&paginacao=t\&paginaAtual=1 >. doi: $16328-1$.

SILVA, J.; JUCKSCH, I.; FERES, C. I. M. A.; TAVARES, R. C. de. Fauna do solo em sistemas de manejo com café. Journal of Biotechnology and Biodiversity, v. 3, n. 2, p. $\quad 59-71, \quad 2012 . \quad$ Disponível em: <http://revista.uft.edu.br/index.php/JBB/article/view/263/183>. doi: 2179-4804.

SILVA, R. F. da.; CORASSA, G. M.; BERTOLLO, G. M.; SANTI, A. L.; STEFFEN, R. $B$. Fauna edáfica influenciada pelo uso de culturas e consórcios de cobertura do solo. ISSN 1983-4063 - Pesquisa Agropecuária Tropical, Goiânia, v. 43, n. 2, p. 130-137, 2013. Disponível em: <http://dx.doi.org/10.1590/S198340632013000200001>. doi: 10.1590/S1983-40632013000200001.

SILVA, R. F. da.; AQUINO, A. M. de.; MERCANTE, F. M.; GUIMARÃES, M. de F. Macrofauna invertebrada do solo em sistema integrado de produção agropecuária no Cerrado. Acta Sci. Agron, Maringá, v. 30, supl., p. 725-731, 2008. Disponível em: <http://www.redalyc.org/articulo.oa?id=303026583018>. doi: 10.4025/actasciagron.v30i5.5974.

SILVA, R. F. da; AQUINO, A. M. de.; MERCANTE, F. M.; GUIMARÃES, M. de F. Macrofauna invertebrada do solo sob diferentes sistemas de produção em Latossolo da Região do Cerrado. Pesquisa Agropecuária Brasileira, Brasília, v. 41, n. 4, p. 697-704, 2006. Disponível em: <http://dx.doi.org/10.4025/actasciagron.v30i5.5974>. doi: 10.4025/actasciagron.v30i5.5974.

SOUZA, R. C. de.; CORREIA, M. E. F.; PEREIRA, M. G.; SILVA, E. M. R. da.; PAULA, R. R.; MENEZES, L. F. T. de. Estrutura da comunidade da fauna edáfica em fragmentos florestais na Restinga da Marambaia, RJ. Revista Brasileira de Ciências Agrárias, v. 3, n. 1, p. 49-57, 2008. Disponível em: <https://www.alice.cnptia.embrapa.br/alice/handle/doc/629940?mode=full>. $\quad$ doi: 629940.

VICENTE, N. M. de F.; CURTINHAS, J. N.; PEREZ, A. L.; PREZOTTI, L. Fauna Edáfica Auxiliando a Recuperação de Áreas Degradadas do Córrego Brejaúba, MG. Floresta e Ambiente, p. 104-110, 2010. Disponível em < http://www.floram.org/files/v17n2/v17n2a5.pdf>. doi: 10.4322/floram.2011.013.

WINK, C.; GUEDES, J. V. C.; FAGUNDES, C. K.; ROVEDDER, A. P. Insetos edáficos como indicadores da qualidade ambiental. Revista de Ciências 
Agroveterinárias, Lages, v. 4, n. 1, p. 60-71, 2005. Disponível em: <http://rca.cav.udesc.br/rca_2005_1/wink.pdf> doi: 1676-9732. 